\title{
Enhancing HIV prevention and care among men who have sex with men (MSM): Insights from social psychology
}

\author{
Rusi Jaspal \& Matthew Page
}

Men who have sex with men (MSM) are a key population in the HIV epidemic in Western industralised societies. Significant strides have been made in preventing HIV infection in MSM - indeed, a 60 per cent decrease in HIV incidence was observed in London at the end of 2016 [1]. This can be attributed to the combined effect of treatment as prevention and pre-exposure prophylaxis (PrEP) in high-risk MSM. However, sexual risk-taking, inconsistent condom use, and low uptake of PrEP remain obstacles to eradicating new HIV transmissions. Advances have also been made in enhancing HIV care. In England, 87\% of MSM living with HIV have been diagnosed and over $90 \%$ are now on effective ART [2]. Yet, some patients struggle to accept and adjust to their HIV diagnosis. Some decide not to initiate ART. Some are lost to followup. Often, the underlying causes are psychosocial in nature. We believe that social psychology has a role to play in developing steps to improve HIV prevention efforts and patient engagement with HIV care.

Psychosocial constructs, such as identity, culture and psychological wellbeing, are central to effective prevention and care. Identity Process Theory [3] postulates that individuals regulate their sense of identity by attempting to deflect threats to important 'principles' of identity, such as self-esteem and sense of continuity. It is easy to see how diagnosis with HIV could challenge one's sense of continuity over time, or how exposure to HIV stigma could threaten self-esteem. Our culture, in part, shapes the meanings we attach to events and situations, which in turn determines the extent they enhance or threaten the identity principles. For instance, HIV will be more 
stigmatised in some cultural contexts than in others. In response to threats to identity, we engage in coping strategies. Some are maladaptive and doomed to failure, while others are proactive and effective in the long-term. Psychological wellbeing can be defined as the equilibrium between adverse events and situations and our capacity to cope with them effectively. After all, adverse events do occur. But our capacity to cope effectively depends on the availability of effective coping strategies.

Among MSM, there appears to be a high prevalence of specific psychosocial stressors, which can pose significant threats to identity. Examples include childhood sexual abuse (CSA), homophobia, and HIV stigma. CSA is up to 4 times more prevalent in MSM than in heterosexual men and HIV-positive MSM are more likely than HIVnegative MSM to report a history of CSA [4]. History of CSA is associated with engagement in sexual risk behaviours and, in the case of HIV patients, with disengagement from care. Life-long exposure to heteronormativity and homophobia is prevalent in MSM and is associated with poor mental health outcomes, including anxiety, depression and suicidal ideation [5]. An HIV diagnosis itself is often experienced as a threat to identity given the associated social stigma. In the 2015/16 Stigma Survey it was found that around half of HIV-positive MSM reported negative emotions, such as shame and guilt [6]. HIV stigma can severely undermine self-esteem, induce emotion dysregulation, and lead to disengagement from care and poor adherence to ART [7].

In response to psychological adversity, some MSM may resort to maladaptive coping strategies, such as sexual compulsivity, engagement in chemsex, and unprotected anal intercourse. All of these behaviours aim to reduce threat in one way or another but they can actually undermine sexual health outcomes. Sexual 
compulsivity is predicted by emotion dysregulation, internalised homophobia and problematic attachment styles [8]. Sexual compulsivity is more prevalent in MSM than in heterosexual men, and more prevalent in HIV-positive than in HIV-negative MSM [9]. Various studies highlight a high prevalence of 'chemsex' (drug use in sexualised settings) in MSM, and particularly in those living with HIV, which is associated with diagnosed depression or anxiety and the desire for escapism [10]. Unprotected anal intercourse may reflect a desire for intimacy and acceptance from others, especially in response to long-standing lack of intimacy, and to rejection and exclusion [11]. It is easy to see how all of these practices can increase the risk of HIV infection in uninfected MSM and lead to poor sexual health outcomes in HIV-infected MSM. Homophobia and HIV stigma, in particular, may contribute to disengagement from HIV prevention, such as PrEP, and HIV care, namely ART. Some MSM may not wish to view themselves as 'high-risk' given the stigma appended to this category [12]. Imagery that emphasises particular sexual behaviours or 'promiscuous' lifestyles may lead some MSM to disengage from PrEP and to view it as something beneficial to others rather than to oneself. Conversely, HIV patients may not initiate ART due to its association with a stigmatised chronic condition. Understanding these psychosocial barriers is key. The ways in which clinicians and other practitioners discuss these tools with patients can greatly influence attitudes, acceptability and, ultimately, uptake. Use of nonstigmatising, culturally sensitive language is essential.

It is vital to acknowledge the significant psychosocial changes that often accompany an HIV diagnosis. The patient may respond by denying the reality of his infection and, to support the denial strategy, he may disengage from care, refuse to initiate ART, and engage in escapist behaviours that facilitate disconnection from his 
reality [3]. All of these practices are maladaptive and can lead to poor patient outcomes and to onward HIV transmission. Conversely, some patients may begin to prepare themselves psychologically (that is, to change their mindset, their social circles) in anticipation of a threat, e.g. being categorised as high-risk, or receiving a positive HIV diagnosis. We might refer to this as 'anticipatory re-structuring'. Some may re-conceptualise the meaning of their HIV infection, possibly viewing it as a positive 'turning-point' or a 'wake-up call'. This in turn can facilitate engagement with care, a more health-conscious lifestyle and increased engagement with others living with HIV. Some patients seek acceptance from and inclusion in groups and networks that can offer social support, such as an HIV support group or a PrEP advocacy group. Involvement in these groups can challenge the stigma they may have internalised and offer novel, affirmative ways of thinking about themselves.

Practitioners involved in the care of MSM at risk of, or living with, HIV can intervene to promote effective strategies for coping with psychosocial stressors. The first author has recently written a book [3] which outlines the ways in which practitioners can identify potential risk factors for adopting maladaptive coping strategies, such as chemsex and unprotected sex, and how they can increase patient access to proactive strategies, such as anticipatory re-structuring, and the derivation of social support. Practitioners may be able to predict coping strategies in patients, which in turn can enable them to put into practice appropriate methods for preventing adverse patient outcomes. These may include specific support with CSA, substance misuse, or homophobia. Moreover, the book highlights how effective clinical interventions, such as PrEP or ART uptake, may actually be experienced as threatening for identity and, thus, rejected by patients who could benefit from them clinically. This 
has much to do with language, identity and culture and those practitioners cognisant of these factors will be more effective in their practice. The gulf between biomedical and psychosocial approaches to HIV prevention and care must be bridged. This editorial is an attempt to do just that.

\section{REFERENCES}

[1] Brown, AE, Mohammed, H, Ogaz, D et al. Fall in new HIV diagnoses among men who have sex with men (MSM) at selected London sexual health clinics since early 2015: testing or treatment or pre-exposure prophylaxis (PrEP)?. Euro Surveillance. 2017; 22: 30553

[2] Brown AE, Kirwan PD, Chau C, Khawam J, Gill ON, Delpech VC. Towards elimination of HIV transmission, AIDS and HIV-related deaths in the UK - 2017 report. November 2017, Public Health England, London.

https://assets.publishing.service.gov.uk/government/uploads/system/uploads/attach ment data/file/675809/Towards elimination of HIV transmission AIDS and HIV rel ated deaths in the UK.pdf

[3] Jaspal R. Enhancing Sexual Health, Self-Identity and Wellbeing among Men Who Have Sex With Men: A Guide for Practitioners. London: Jessica Kingsley Publishers; 2018.

[4] Lloyd S, Operario D: HIV Risk among Men Who Have Sex With Men Who Have Experienced Childhood Sexual Abuse: Systematic Review and Meta-Analysis. AIDS Education \& Prevention. 2012, 24: 228-241. 10.1521/aeap.2012.24.3.228.

[5] Walch SE, Ngamake ST, Bovornusvakool W, Walker SV. Discrimination, internalized homophobia, and concealment in sexual minority physical and mental health. Psychology of Sexual Orientation and Gender Diversity. 2016, 3: 37-48. [6] HIV in the UK: Changes and Challenges; Actions and Answers The People Living With HIV Stigma Survey UK 2015, Men Who Have Sex With Men (MSM).

http://stigmaindexuk.org/reports/2016/MSM.pdf

[7] Jeffries WL, IV, Townsend ES, Gelaude DJ, Torrone EA, Gasiorowicz M, Bertolli J. HIV stigma experienced by young men who have sex with men (MSM) living with HIV infection. AIDS Education \& Prevention. 2015;27(1):58-71.

[8] Pachankis JE, Rendina HJ, Restar A, Ventuneac A, Parsons JT. A minority stressemotion regulation model of sexual compulsivity among highly sexually active gay and bisexual men. Health Psychology. 2015, 34(8):829-40. doi: 10.1037/hea0000180. [9] Coleman E, Horvath KJ, Miner M et al. Compulsive sexual behavior and risk for unsafe sex among Internet using men who have sex with men. Archives of Sexual Behavior. 2010;39(5):1045-1053

[10] Bourne A, Reid D, Hickson F, Torres Rueda S, Weatherburn P. The Chemsex study: drug use in sexual settings among gay \& bisexual men in Lambeth, Southwark \& Lewisham. London: Sigma Research, London School of Hygiene \& Tropical Medicine; 2014. www.sigmaresearch.org.uk/chemsex 
[11] Starks TJ, Payton G, Golub SA, Weinberger CL, Parsons JT. Contextualizing condom use: Intimacy Interference, stigma, and unprotected sex. Journal of Health Psychology. 2014;19(6):711-720. doi:10.1177/1359105313478643.

[12] Jaspal R, Daramilas C. Perceptions of pre-exposure prophylaxis (PrEP) among HIVnegative and HIV-positive men who have sex with men (MSM). Cogent Medicine 2016;3:1-16. 